\title{
Polarization Dispersion Characteristics of Propagation Paths in Urban Mobile Communication Environment
}

Tetsuro IMAI $^{\dagger \mathrm{a})}$ and Koshiro KITAO ${ }^{\dagger}$, Members

\begin{abstract}
SUMMARY In order to employ Multiple-Input-Multiple-Output (MIMO) techniques, multiple antenna branches are necessary and as a consequence the installation space requirements are increased. Since the installation space is limited, much attention is now focused on utilizing polarization characteristics in MIMO configurations to relax the requirements. This is called Orthogonal Polarization-MIMO in this paper. To evaluate accurately the performance of Orthogonal Polarization-MIMO, a channel model that can handle the polarization dispersion characteristics of propagation paths is essential. Up to now, the spatial-temporal dispersion characteristics of paths have been investigated in detail. However, there are only a few reports on the polarization dispersion characteristics. In this paper, we propose a new power profile for the rotational polarized angle as an evaluation model for polarization dispersion, and clarify the analyzed power profile based on measurement data in an urban macrocell environment.
\end{abstract}

key words: MIMO, polarization, propagation model, mobile propagation

\section{Introduction}

Multiple-Input-Multiple-Output (MIMO) transmission techniques have been actively investigated in wide-band mobile communication systems to achieve higher transmission rates. These techniques utilize multiple orthogonal channels and require antenna branches on both the transmission and reception sides, where the number of antenna branches is decided according to the required transmission rates. The branches must be installed while maintaining the proper spacing in order to maintain orthogonality between channels [1]-[4]. Therefore, the installation space requirements increase when the number of branches increases. This imposes considerable constraints on introducing MIMO techniques to actual mobile communication systems. To address this, much attention is now focused on utilizing polarization characteristics in MIMO configurations [5]-[7]. We call this the Orthogonal Polarization-MIMO technique.

In order to evaluate the performance of Orthogonal Polarization-MIMO, the polarization dispersion characteristics of propagation paths between the transmitter (Tx) and receiver $(\mathrm{Rx})$ are needed in addition to the spatial-temporal dispersion characteristics [2], [5]-[7]. In general, polarization characteristics have been evaluated using the CrossPolarization Power Ratio (XPR) as an evaluation indicator. Up to now, there have been only a few reports on the XPR characteristics of propagation paths in macro- and microcel-

\footnotetext{
Manuscript received January 21, 2013.

Manuscript revised May 20, 2013.

${ }^{\dagger}$ The authors are with NTT DOCOMO INC., Yokosuka-shi, 239-8536 Japan.

a)E-mail: imait@nttdocomo.co.jp

DOI: 10.1587/transcom.E96.B.2380
}

lular environments, even though the XPR characteristics of a narrowband channel have been actively investigated [3], [8]. We experimentally investigated the XPR characteristics of the paths in a macrocellular environment and showed the obtained results in [9]. However, the characteristics were not modeled. In [10], an angular-delay power spectrum density channel model was proposed. Since the model includes full information of the polarization as polarimetric complex path weights, we can estimate the polarization dispersion characteristics. Here, the values for model parameters are given for each measurement street; however, the behavior of the parameter values over all measurement streets is not modeled.

In [11], the XPR characteristics of paths were experimentally investigated in an indoor environment and a model for the XPR distribution based on a cluster was proposed. We believe that this modeling method is not the best because the relationship between power and the XPR of a path is not clear. When modeling the polarization dispersion characteristics of paths, the XPR is not suitable as an evaluation indicator. In this paper, we define the rotational polarized angle as an evaluation indicator and propose a new power profile for the rotational polarized angle as an evaluation model for polarization dispersion [12], [13]. We then clarify the actual power profile by analyzing measurement data in an urban macrocellular environment. Hereafter, we refer to the power profile as the Power Polarization Profile or PPP.

\section{Evaluation Model of Polarization Dispersion}

In general, propagation paths between the Tx and Rx are characterized by the time of arrival (TOA), angle of departure (AOD), and angle of arrival (AOA). Up to now, power distributions with respect to the parameters have been theoretically and experimentally investigated and they have been modeled as power profiles with respect to these parameters [3]. As shown in Fig. 1, a vertically (or horizontally)polarized wave transmitted from a base station (BS) arrives at a mobile station (MS), changing its rotational polarized angle due to reflection, diffraction, and scattering from the surrounding structures, e.g., buildings, and vice versa. Therefore, the rotational polarized angle at the reception point differs for each path. Taking these into consideration, using the rotational polarized angle as an indicator is more natural than using the XPR when evaluating the polarization characteristics of a path. The proposed polarization model is described in detail hereafter. 


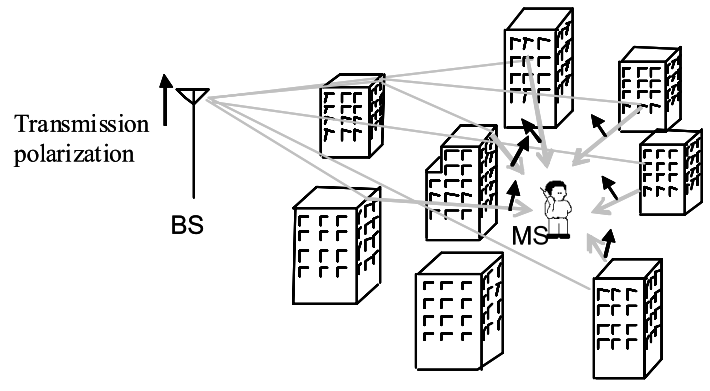

Fig. 1 Relationship between transmission and reception polarization in an urban mobile communication environment.

Let $P_{n}^{(H)}$ and $P_{n}^{(V)}$ be the power of the $n$-th path received at a horizontal polarized antenna and a vertical polarized antenna, respectively. In the model, the rotational polarized angle of the $n$-th path, $\theta_{n}$, is defined as

$$
\theta_{n}=\tan ^{-1}\left(P_{n}^{(H)} / P_{n}^{(V)}\right) .
$$

The range of $\theta_{n}$ is $[0,90]$ in degrees because the values of $P_{n}^{(H)}$ and $P_{n}^{(V)}$ are never negative values. The $\theta_{n}$ of 0 means that the wave through the $n$-th path is vertically polarized at the reception point, and the $\theta_{n}$ of 90 degrees means that the wave through the $n$-th path is horizontally polarized at the reception point. The received power of the $n$-th path, $P_{n}$, is given by $P_{n}^{(H)}+P_{n}^{(V)}$. We define the envelope of the distribution of $P_{n}$ with respect to $\theta_{n}$ as the PPP.

The spread for $\theta_{n}$ can be defined similarly to a delay spread and angle spread. We call this new spread a polarization spread. Letting $P(\theta)$ be the power distribution function of the rotational polarized angle, the polarization spread, $\sigma_{\theta}$, is expressed as

$$
\sigma_{\theta}=\sqrt{\int_{0}^{\frac{\pi}{2}}\left(\theta-m_{\theta}\right)^{2} P(\theta) d \theta / \int_{0}^{\frac{\pi}{2}} P(\theta) d \theta},
$$

where $m_{\theta}$ is the mean value of $\theta_{n}$ expressed as

$$
m_{\theta}=\int_{0}^{\frac{\pi}{2}} \theta \cdot P(\theta) d \theta \mid \int_{0}^{\frac{\pi}{2}} P(\theta) d \theta .
$$

Note that, in order to evaluate the polarization spread with respect to the vertically (or horizontally) polarized direction at the time of transmission, we treat the value of $m_{\theta}$ as 0 and 90 degrees for vertical and horizontal transmissions, respectively, in this paper.

When assuming the power distribution function of the rotational polarized angle, the XPR of a narrow band channel can be easily given by

$$
X P R=\int_{0}^{\frac{\pi}{2}} P(\theta) \cos (\theta) d \theta \mid \int_{0}^{\frac{\pi}{2}} P(\theta) \sin (\theta) d \theta .
$$

This is one of the advantages of the proposed model, because the XPR cannot be expressed from the above mentioned model based on the distribution of the XPR of the paths [11].
Table 1 Experimental conditions.

\begin{tabular}{|c|c|c|c|}
\hline Investigation area & Yoyogi & Aoyama & Yokohama \\
\hline Transmission power & \multicolumn{3}{|c|}{$2.2255 \mathrm{GHz}$} \\
\hline Frequency & \multicolumn{3}{|c|}{$30 \mathrm{Mbps}$} \\
\hline Bit rate & \multicolumn{3}{|c|}{$\begin{array}{c}\text { Sleeve antenna for V-polarized wave } \\
\text { Slotted cylinder antenna for H-polarized wave }\end{array}$} \\
\hline Txantenna & $150 \mathrm{~m}$ & $60 \mathrm{~m}$ & $40 \mathrm{~m}$ \\
\hline Txantenna height & Eight dual (V and H)-polarized sub-array antennas \\
\hline are vertically aligned
\end{tabular}

\section{Measurement}

\subsection{Measurement System}

Table 1 gives the primary experimental conditions. The measurement system is constructed around a steppingcorrelator radio channel sounder, which is based on the popular pseudo noise (PN) correlation method [14]. The system utilizes the maximum length sequence with a period of 1023 bits as the PN sequence. At the transmitter, a 2.2255$\mathrm{GHz}$ carrier is BPSK modulated using a 30-Mbps PN sequence and radiated from the Tx antenna. At the receiver, 16-complex impulse responses at maximum are obtained at approximately the same time by switching the input ports connected to the antenna elements at high speed. The time resolution of the impulse responses is approximately $33 \mathrm{~ns}$.

For the measurements, as the Tx antenna, a sleeve antenna and a slotted cylinder antenna are used to transmit vertically and horizontally polarized waves, respectively. The directional radiation pattern and the top gain of both antennas are almost identical (See Appendix). The top gain is approximately $2.2 \mathrm{dBi}$. The $\mathrm{Rx}$ antenna has eight dual ( $\mathrm{V}$ and $\mathrm{H})$-polarized sub-array antennas that are vertically aligned with the spacing of $0.5 \lambda$. Here $\lambda$ denotes the wavelength. Each sub-array antenna has two feeding ports to receive the vertically and horizontally polarized waves, respectively. The directional radiation pattern and the top gain for both polarizations are almost identical. The vertical and horizontal 3-dB beamwidth are $110 \mathrm{deg}$. and $30 \mathrm{deg}$., respectively, and the top gain is $8.29 \mathrm{dBi}$. We describe the Rx antenna configuration and its radiation patterns in detail in the Appendix.

\subsection{Investigation Environment}

We conducted the measurements in three urban areas in Tokyo (Yoyogi and Aoyama) and Kanagawa (Yokohama), Japan. Figure 2 shows the investigation areas. The Tx antenna is mounted on the rooftop of a building as a BS antenna, and the Rx antenna is mounted on a roof of a measurement wagon as an MS antenna. The Tx antenna height 


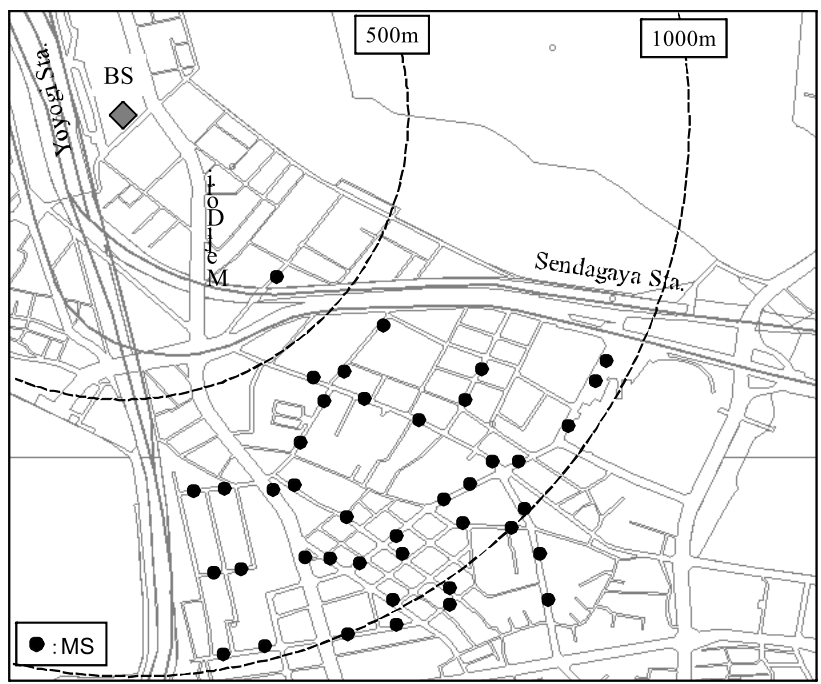

(a)

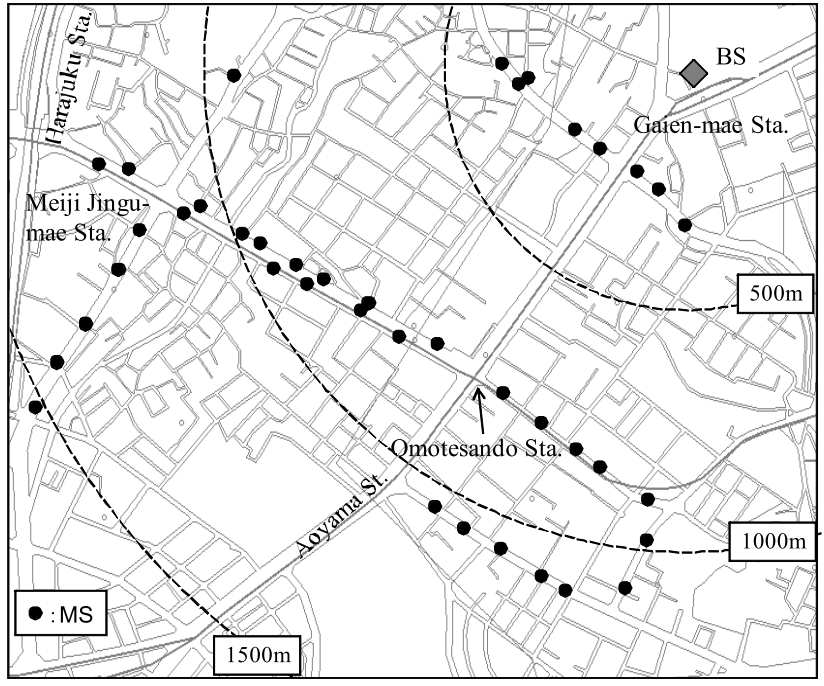

(b)

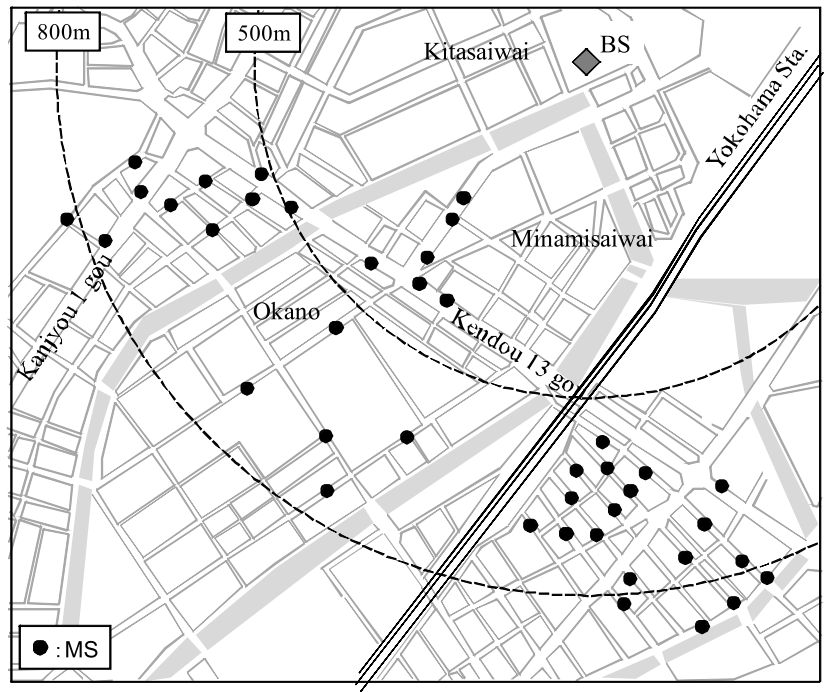

(c)

Fig. 2 Investigation areas. (a) Yoyogi, (b) Aoyama, (c) Yokohama. and the $\mathrm{Rx}$ antenna height are given in Table 1 . The average building height is approximately $20 \mathrm{~m}$ in all the investigation areas. Measurements are conducted while the wagon is stopped beside a regular street. At each reception point (or MS location), the Rx antenna is rotated in the horizontal plane and 24-azimuth directional (angle interval: $15 \mathrm{deg}$.) data points are obtained. The number of snapshots of the complex impulse response at each input port for each direction is 20 . We first obtained data when transmitting a vertically polarized wave from the sleeve antenna and next obtained data when transmitting a horizontally polarized wave from the slotted cylinder antenna. Here, we manually changed the sleeve antenna to the slotted cylinder antenna because the measurement system is a single input multiple output (SIMO) measurement system.

As indicated in Table 1 and Fig. 2, there are 40 or 41 (non-line-of-sight) reception points, the distances of which from the transmission point (or BS location) are distributed within approximately $1 \mathrm{~km}$ (see Table 1 for details).

\subsection{Data Processing}

The data processing procedure in order to extract the arrival paths for each MS location is described below.

First, the average power delay profile is calculated from 20 (snapshots) $\times 8$ (Rx sub-array antennas) measured complex impulse responses for each Rx antenna azimuth direction (hereafter referred to as azimuth directional bin) and each polarization combination, i.e., $(\mathrm{Tx}$ Pol., $\mathrm{Rx}$ Pol. $)=(\mathrm{V}$, $\mathrm{V}),(\mathrm{H}, \mathrm{V}),(\mathrm{V}, \mathrm{H})$, and $(\mathrm{H}, \mathrm{H})$. Next, the total power delay profile is calculated by adding 24 (azimuth directional bins) $\times 4$ (polarization combinations) power delay profiles. The peaks are detected in the overall power delay profile. In this process, the peak that satisfies the following conditions is disregarded because it is doubtful that such a peak is actually a propagation path.

- The delay time is within $\pm 33 \mathrm{~ns}$ around the higher peaks.

- The power ratio to the highest peak is less than $-30 \mathrm{~dB}$.

- The power ratio to the noise level is within $5 \mathrm{~dB}$.

Each obtained peak is recognized to be the delay time of a cluster of paths.

The arrival paths are obtained by detecting peaks in the three-dimensional power angle profile at the delay time of the cluster. This is achieved as described hereafter. First, 20 elevation power angle profiles at the delay time of the cluster are calculated from the 20 measured complex impulse responses for an azimuth directional bin and a polarization combination by applying conventional beamforming [15] with eight Rx sub-array antennas, and then they are averaged. Next, the three-dimensional power angle profile is calculated by joining the 24-azimuth-directional bins and adding four polarization combinations. The final procedure for detecting the peaks as paths is described below.

1. Project the three-dimensional power angle profile in the 
horizontal plane in order to obtain the azimuth power angle profile.

2. Select the azimuth directional bins by detecting the peaks in the azimuth power angle profile. Here, the peaks for which the power ratio to the highest peak is less than $-20 \mathrm{~dB}$ are disregarded.

3. Detect the peaks as the paths in the elevation power angle profile for each selected azimuth directional bin. Here, the peaks for which the power ratio to the highest peak is less than $-10 \mathrm{~dB}$ are disregarded.

Here, the azimuth angle of each path is the angle corresponding to the azimuth directional bin that includes the path.

As a result, the path power for each polarization combination is obtained. Hereafter, this paper uses the following notations.

- $P_{n}^{(B S: X, M S: Y)}$ is the power of the $n$-th path where $X$ is the transmission polarization at the $\mathrm{BS}$ and $Y$ is the reception polarization at the MS.

- $P_{n}^{(B S: X)}$ is the total power of the $n$-th path for the $X$ transmission polarization, i.e., $P_{n}^{(B S: X, M S: V)}+$ $P_{n}^{(B S: X, M S: H)}$

- $P_{n}^{(M S: Y)}$ is the total power of the $n$-th path for the $Y$ reception polarization, i.e., $P_{n}^{(B S: V, M S: Y)}+P_{n}^{(B S: H, M S: Y)}$

- $\sigma_{\theta}^{(B S: X)}$ is the polarization spread for the $X$ transmission polarization.

- $\sigma_{\theta}^{(M S: Y)}$ is the polarization spread for the $Y$ reception polarization.

- $X P R_{n}^{(B S: X)}$ is the XPR of the $n$-th path for the $X$ transmission polarization.

Here, with these parameters, the total power at the MS, $P_{\text {total }}$, is expressed as

$$
\begin{aligned}
P_{\text {total }}= & \sum_{n} P_{n}^{(B S: V, M S: V)}+P_{n}^{(B S: V, M S: H)}+P_{n}^{(B S: H, M S: V)} \\
& +P_{n}^{(B S: H, M S: H)}
\end{aligned}
$$

and the XPR of a narrowband channel for the $X$ transmission polarization, $X P R^{(B S: X)}$, is expressed as

$$
X P R^{(B S: X)}=\sum_{n} P_{n}^{(B S: X, M S: X)} / \sum_{n} P_{n}^{(B S: X, M S: Y)} .
$$

From our measurements, the PPP of four types

- PPP when transmitting with vertical polarization,

- PPP when transmitting with horizontal polarization,

- PPP when receiving with vertical polarization,

- PPP when receiving with horizontal polarization.

is obtained. Here when reception polarization is fixed as $Y$ (=V or $H$ ), the rotational polarized angle of a path is defined by

$$
\theta_{n}=\tan ^{-1}\left(P_{n}^{(B S: V, M S: Y)} / P_{n}^{(B S: H, M S: Y)}\right) .
$$

\section{Polarization Dispersion Characteristics}

In this section, we describe the polarization characteristics based on the analysis results of the measurement data. Note that the cumulative $50 \%$ value of the number of paths at each MS is 90 (Yoyogi), 168 (Aoyama), and 157 (Yokohama).

\subsection{Cross-Polarization Power Ratio}

Figure 3 shows the cumulative distributions of $X P R^{(B S: V)}$ in Yoyogi, Aoyama, and Yokohama assuming a narrowband channel. The distribution is approximately recognized as a Gaussian distribution and the cumulative $50 \%$ value is approximately $8 \mathrm{~dB}$, which is a typical value in an urban area based on [3] and [8]. Figure 4 shows the cumulative distributions for the XPR of paths, $X P R_{n}^{(B S: V)}$, where the results from all MS locations are plotted. From this figure, we understand that the cumulative $50 \%$ value is approximately $3 \mathrm{~dB}$ and the attribute is significantly different than that for $X P R^{(B S: V)}$. We believe that this difference depends on the power of each path. Figure 5 shows the relationship between $X P R_{n}^{(B S: V)}$ and $P_{n}^{(B S: V)}$ in Aoyama. Here the value of $P_{n}^{(B S: V)}$ is normalized by $P_{\text {total }}$ at each MS location. From Fig. 5, we find that there is a tendency. The received power increases when the XPR is larger. It is however difficult to model this relationship.

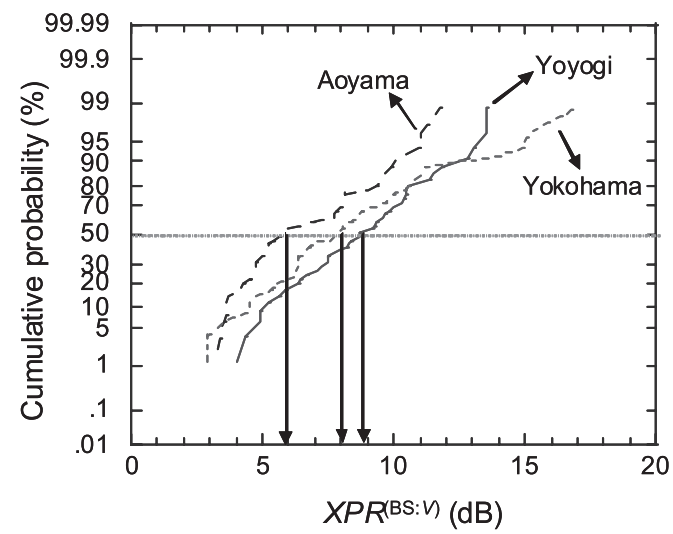

Fig. 3 Cross-polarization power ratio of narrowband channel when transmitting with vertical polarization.

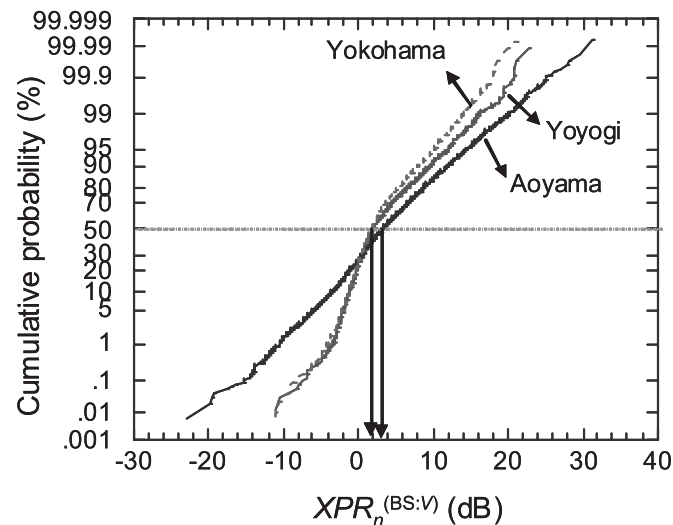

Fig. 4 Cross-polarization power ratios of paths when transmitting with vertical polarization 


\subsection{Evaluation Results Based on Proposed Model}

Figure 6 shows the power polarization profiles in the

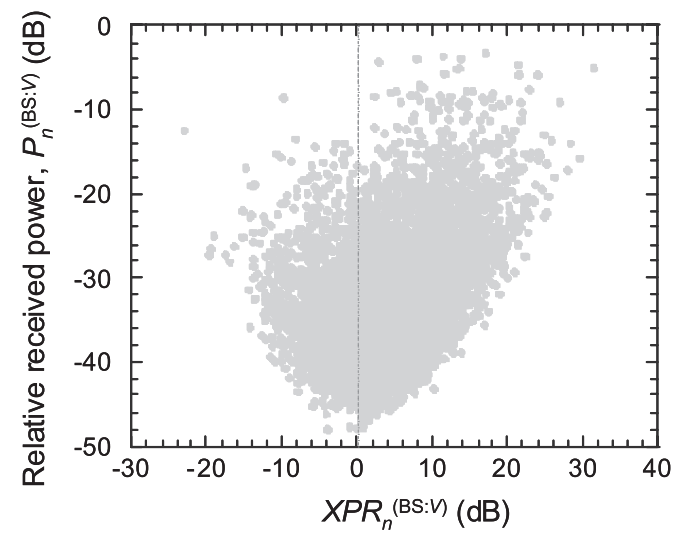

Fig. 5 Relationship between received power and cross-polarization power ratio for each path in Aoyama area when transmitting with vertical polarization.
Aoyama area, where the results from all MS locations are counted. Note that each profile was obtained as follows.

1. The received power of each path was normalized based on $P_{\text {total }}$ at each location.

2. The normalized power was moving-integrated over $1 \mathrm{deg}$. in $0.1 \mathrm{deg}$. steps.

3. The integrated value is normalized by the maximum value.

From these results, we find that the distribution of the rotational polarized angle, $\theta_{n}$, has a peak of $0 \mathrm{deg}$. when the transmission/reception polarization is vertical. When the transmission/reception polarization is horizontal, the peak is $90 \mathrm{deg}$. We confirmed that the characteristics of the distribution in Yoyogi and Yokohama are the same. This means that we can easily characterize the polarization dispersion by using the rotational polarized angle as an indicator.

Figure 7 shows the cumulative distribution of the polarization spread calculated at each MS location, which suggests the spatial dispersion characteristics of the spread. The mean value and the standard deviation of the polarization spread are shown in Table 2. The mean value of the spread

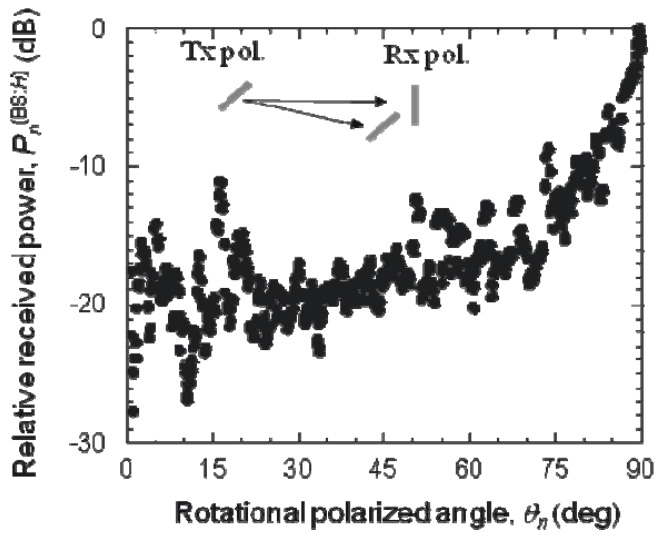

(b)

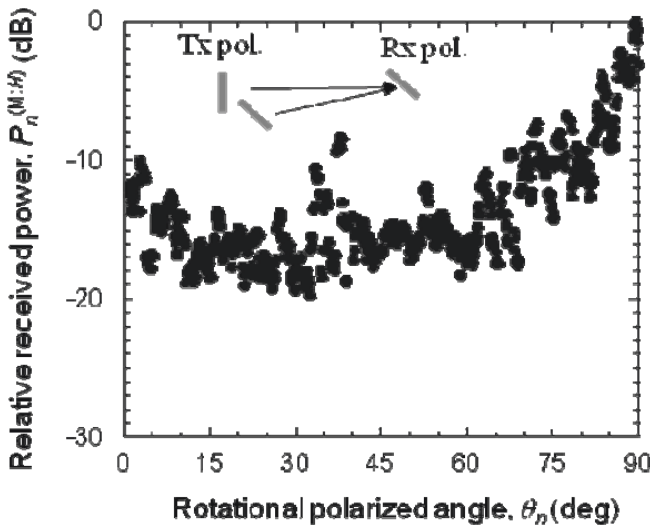

(d)

Fig. 6 Power polarization profile (a) When transmitting with vertical polarization, (b) when transmitting with horizontal polarization, (c) when receiving with vertical polarization, (d) when receiving with horizontal polarization. 


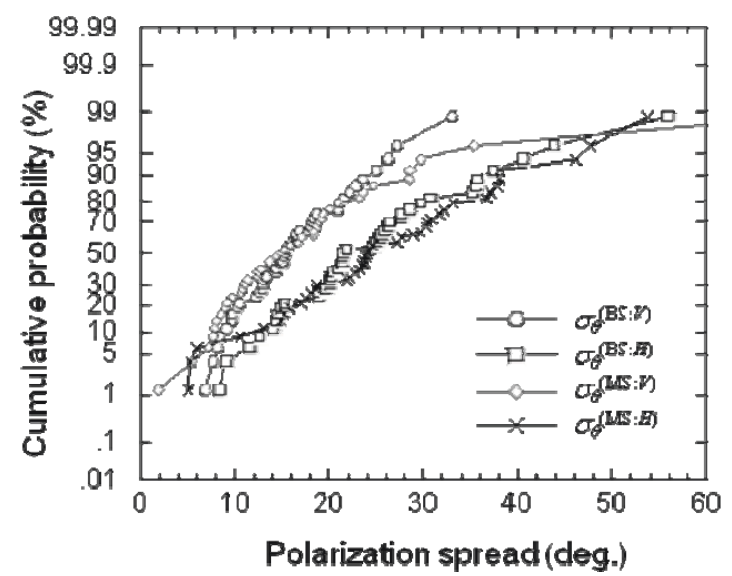

(a)

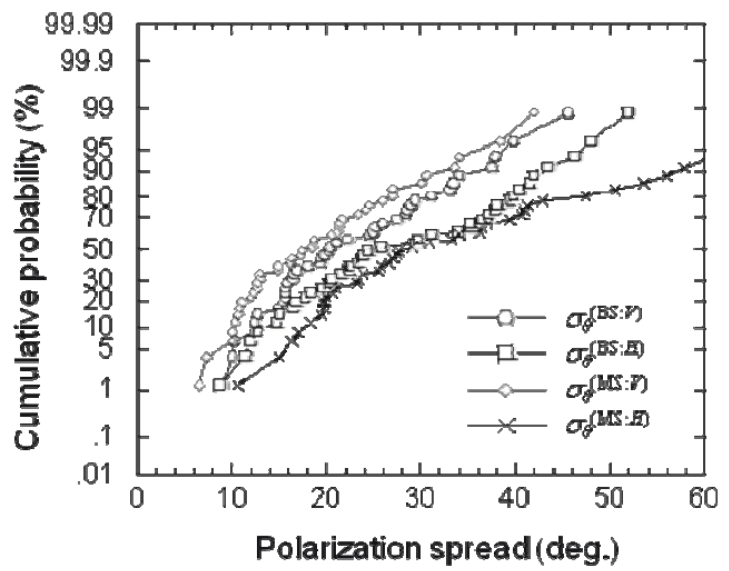

(b)

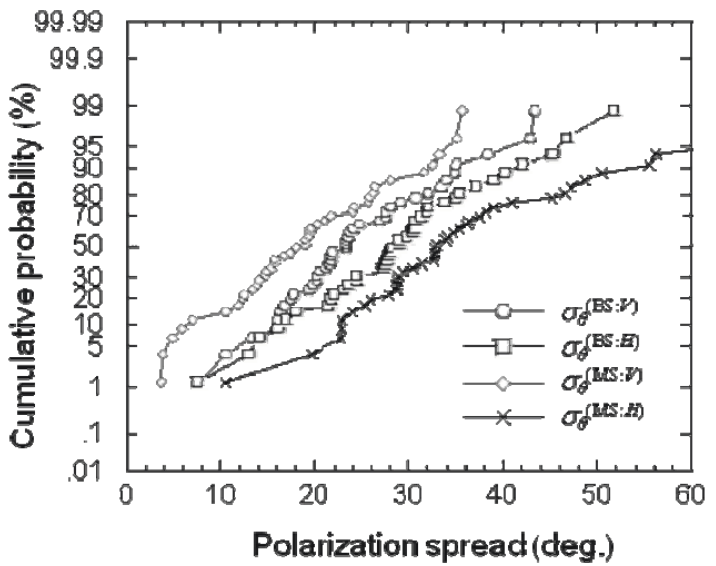

(c)

Fig. 7 Cumulative distribution of polarization spread. (a) Yoyogi, (b) Aoyama, (c) Yokohama.

increases in the order of Yoyogi, Aoyama, and Yokohama. We expect that this behavior depends on the BS antenna height. The lower the BS antenna height, the probability increases that the polarization direction changes due to reflection, diffraction, and scattering from the surrounding struc-
Table 2 Polarization spread.

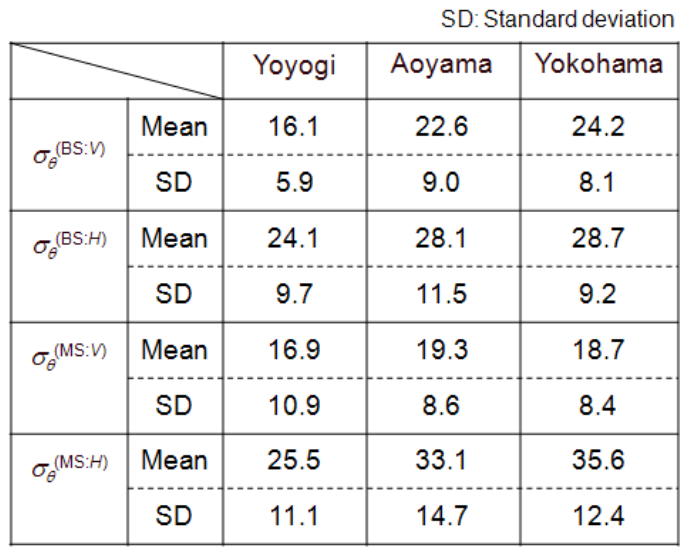

tures. On the other hand, we cannot find a tendency between the areas with respect to the standard deviation. It is thought that the relationship between the standard deviation of the polarization spread and the BS antenna height is weak unlike the case for the mean value.

\section{Formulation of Power Polarization Profile}

In this section, we formulate the PPP. Figure 8 shows the regression results of the profiles shown in Figs. 6(a) and (b), where the function is assumed as

$$
P(\theta)=a \exp \left(-\left|\theta-m_{\theta}\right| / b\right)+c
$$

where $m_{\theta}$ is 0 and 90 degrees for vertical and horizontal transmissions, respectively. In (8), parameters, $a, b$, and $c$, are the regression coefficients. The obtained regression coefficients are shown in Table 3. Note that the regression results for the profiles in Figs. 6(c) and 6(d) are also shown in the table, and $R$ is the correlation coefficient between the sample (i.e. measured PPP) and the regression results. We conclude that the PPP can be modeled by an exponential distribution with a constant term, i.e.,

$$
P(\theta) \propto \frac{1}{\beta} \exp \left(-\left|\theta-m_{\theta}\right| / \beta\right)+\gamma .
$$

Here, $\beta$ is a parameter in the term of the exponential distribution and $\gamma$ represents the constant term.

When assuming (9) as a function of the PPP, the polarization spread for vertical transmission (or vertical reception) is expressed as

$$
\begin{aligned}
\sigma_{\theta} & =\sqrt{\frac{\int_{0}^{\theta_{0}} \theta^{2}\left\{\frac{1}{\beta} \exp (-|\theta| / \beta)+\gamma\right\} d \theta}{\int_{0}^{\theta_{0}}\left\{\frac{1}{\beta} \exp (-|\theta| / \beta)+\gamma\right\} d \theta}} \\
& =\sqrt{\frac{\int_{0}^{\theta_{0}} \theta^{2} e^{-\theta / \beta} d \theta+\beta \gamma \int_{0}^{\theta_{0}} \theta^{2} d \theta}{\int_{0}^{\theta_{0}} e^{-\theta / \beta} d \theta+\beta \gamma \int_{0}^{\theta_{0}} d \theta}} \\
& =\sqrt{\frac{2 \beta^{2}-e^{-\theta_{0} / \beta}\left(\theta_{0}^{2}+2 \beta \theta_{0}+2 \beta^{2}\right)+\frac{\gamma}{3} \theta_{0}^{3}}{1-e^{-\theta_{0} / \beta}+\gamma \theta_{0}}}
\end{aligned}
$$




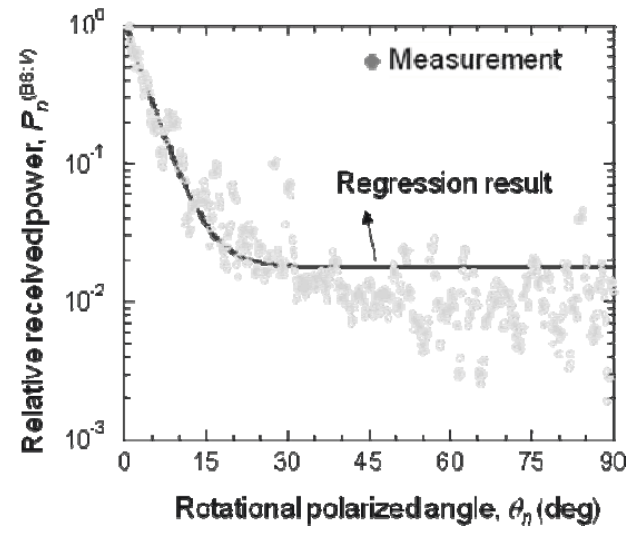

(a)

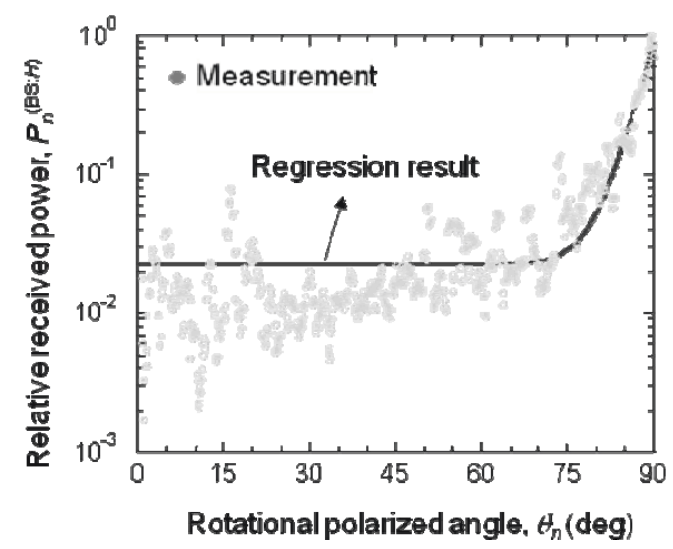

(b)

Fig. 8 Regression results of power polarization profiles. (a) When transmitting with vertical polarization, (b) when transmitting with horizontal polarization.

and the XPR of the narrow band channel for vertical transmission (or vertical reception) is expressed by

$$
\begin{aligned}
X P R & =\frac{\int_{0}^{\theta_{0}} P(\theta) \cos (\theta) d \theta}{\int_{0}^{\theta_{0}} P(\theta) \sin (\theta) d \theta} \\
& =\frac{\int_{0}^{\theta_{0}}\left\{\frac{1}{\beta} \exp (-|\theta| / \beta)+\gamma\right\} \cos (\theta) d \theta}{\int_{0}^{\theta_{0}}\left\{\frac{1}{\beta} \exp (-|\theta| / \beta)+\gamma\right\} \sin (\theta) d \theta} \\
& =\frac{\int_{0}^{\theta_{0}} e^{-\theta / \beta} \cos (\theta) d \theta+\beta \gamma \int_{0}^{\theta_{0}} \cos (\theta) d \theta}{\int_{0}^{\theta_{0}} e^{-\theta / \beta} \sin (\theta) d \theta+\beta \gamma \int_{0}^{\theta_{0}} \sin (\theta) d \theta} \\
& =\frac{1+\beta e^{-\theta_{0} / \beta}-\gamma\left(1+\beta^{2}\right)}{\beta-e^{-\theta_{0} / \beta}-\gamma\left(1+\beta^{2}\right)} .
\end{aligned}
$$

Here, $\theta_{0}$ is $\pi / 2$ in (10) and (11). The relationship between $\sigma_{\theta}$ and the XPR, which is calculated from (10) and (11), is shown in Fig. 9. In the figure, we also show the measurement results when the transmission polarization is vertical in the Aoyama area. We understand that (9) is suitable as the PPP model. Note that in the case of horizontal transmission (or horizontal reception), i.e. $m_{\theta}=90$ degrees, $\sigma_{\theta}$ is identical to (10) and XPR is given by
Table 3 Regression coefficients.

\begin{tabular}{|c|c|c|c|c|}
\hline \multirow{2}{*}{ Nolical } & \multicolumn{2}{|c|}{$\begin{array}{c}\text { Transmission } \\
\text { polarization }\end{array}$} & \multicolumn{2}{c|}{$\begin{array}{c}\text { Reception } \\
\text { polarization }\end{array}$} \\
\cline { 2 - 5 } & Vertical & Horizontal & Vertical & Horizontal \\
\hline$a$ & 0.99 & 0.85 & 1.33 & 0.65 \\
\hline$b$ & 3.7 & 3.0 & 1.5 & 5.2 \\
\hline$c$ & 0.018 & 0.022 & 0.011 & 0.035 \\
\hline$R$ & 0.97 & 0.97 & 0.97 & 0.89 \\
\hline
\end{tabular}

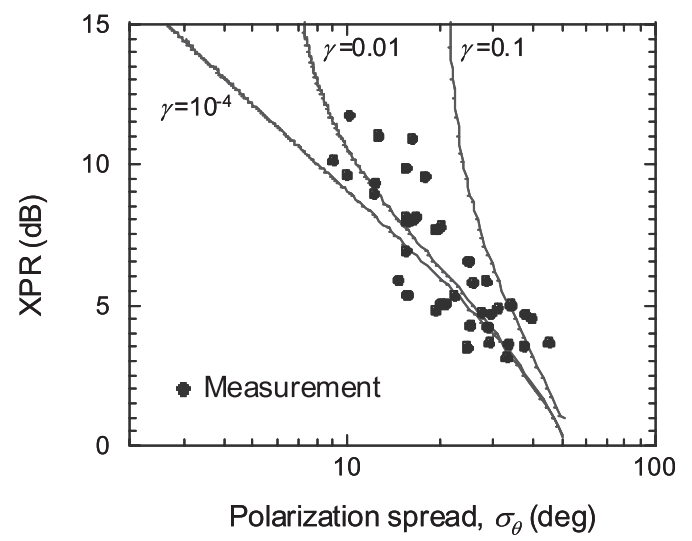

Fig.9 Relationship between $\sigma_{\theta}$ and XPR based on proposed model.

$$
\begin{aligned}
X P R & =\frac{\int_{0}^{\theta_{0}} P(\theta) \cos (\theta) d \theta}{\int_{0}^{\theta_{0}} P(\theta) \sin (\theta) d \theta} \\
& =\frac{\int_{0}^{\theta_{0}}\left\{\frac{1}{\beta} \exp \left(-\left|\theta-\theta_{0}\right| / \beta\right)+\gamma\right\} \cos (\theta) d \theta}{\int_{0}^{\theta_{0}}\left\{\frac{1}{\beta} \exp \left(-\left|\theta-\theta_{0}\right| / \beta\right)+\gamma\right\} \sin (\theta) d \theta} \\
& =\frac{\int_{0}^{\theta_{0}}\left\{\frac{1}{\beta} \exp (-\theta / \beta)+\gamma\right\} \sin (\theta) d \theta}{\int_{0}^{\theta_{0}}\left\{\frac{1}{\beta} \exp (-\theta / \beta)+\gamma\right\} \cos (\theta) d \theta} \\
& =\frac{\beta-e^{-\theta_{0} / \beta}-\gamma\left(1+\beta^{2}\right)}{1+\beta e^{-\theta_{0} / \beta}-\gamma\left(1+\beta^{2}\right)} .
\end{aligned}
$$

\section{Conclusion}

In this paper, in order to evaluate the polarization dispersion characteristics of the propagation paths, we proposed a power polarization profile, which is a power profile for the rotational polarized angle of paths. The main features of this profile are described below.

- The target propagation paths are separated in the time and spatial domains.

- The rotational polarized angle of a path is defined based on the ratio of the received power at the horizontal polarization antenna to that at the vertical polarization antenna.

- The power of a path is the sum of the received powers at the vertical and horizontal polarization antennas.

- The power polarization profile is the averaged power 
distribution with respect to the rotational polarized angle.

Here, the polarization spread, which is the spread for the rotational polarized angle of paths, can be defined similarly to a delay spread and angle spread.

In addition, the power polarization profile and spatial distribution of the polarization spread were analyzed from measurement data for a $2-\mathrm{GHz}$ band in a macrocell scenario in an urban area. Moreover, it was shown that the profile can be formulated by an exponential distribution with a constant term, and the relationship between the polarization spread and the XPR of a narrow band channel was theoretically analyzed based on the formula. The theoretical results agree with the measurement results.

\section{Acknowledgments}

This research was conducted under a research and development contract for radio resource enhancement technologies, organized by the Ministry of Internal Affairs and Communications, Japan.

\section{References}

[1] A. Goldsmith, Wireless Communications, Maruzen \& Cambridge, 2007 (Japanese edition).

[2] C. Chong, F. Watanabe, K. Kitao, T. Imai, and H. Inamura, "Evolution trends of wireless MIMO channel modeling towards IMTAdvanced," IEICE Trans. Commun., vol.E92-B, no.9, pp.27732788, Sept. 2009.

[3] L.M. Correia, ed., Wireless Flexible Personalised Communications, John Wiley \& Sons, 2001.

[4] L.M. Correia, ed., Mobile Broadband Multimedia Networks, Academic Press, 2006.

[5] M. Coldrey, "Modeling and capacity of polarized MIMO channels," VTC2008-Spring, 2008.

[6] K. Cho and Y. Inoue, "Influence of cross-polarization characteristics on indoor MIMO performance using a dual-polarized base station antenna," 2010 APS/URSI Symposium, 2010.

[7] Y. Inoue, K. Komiya, R. Yamaguchi, and K. Cho, "Experimental evaluation of antenna element spacing with mixed polarized MIMO antenna system in LOS environment," EuCAP 2010, 2010.

[8] A. Kainulainen, L. Vuokko, and P. Vainikainen, "Polarization behavior in different urban radio environments at $5.3 \mathrm{GHz}$,' COST273 Temporary Documents, TD(05)018, Italy, Bologna, Jan. 2005.

[9] M. Ghoraishi, G. Ching, N. Lertsirisopon, J. Takada, A. Nishihara, T. Imai, and K. Kitao, "Polar directional characteristics of the urban mobile propagation channel at 2.2 GHz," EuCAP2009, 2009.

[10] K. Sivasondhivat, J. Takada, I. Ida, and Y. Oishi, "Experimental analysis and site-specific modeling of channel parameters at mobile station in an urban macrocellular environment," IEICE Trans. Commun., vol.E91-B, no.4, pp.1132-1144, April 2008.

[11] F. Quitin, C. Oestges, F. Horlin, and P. Doncker, "A polarized clustered channel model for indoor multiantenna systems at $3.6 \mathrm{GHz}$,' IEEE Trans. Veh. Technol., vol.59, no.8, pp.3685-3693, Oct. 2010.

[12] T. Imai and K. Kitao, "Characteristics of polarization dispersion of propagation paths in urban mobile communication environment," IEICE Technical Report, AP2010-147, Jan. 2011.

[13] T. Imai and K. Kitao, "Polarization dispersion characteristics of propagation paths in urban mobile communication environment," VTC2011-Fall, Sept. 2011.

[14] D.C. Cox, "Delay doppler characteristics of multipath propagation at $910 \mathrm{MHz}$ in a suburban mobile radio environment," IEEE Trans Antennas Propag., vol.AP-20, no.5, pp.625-635, Sept. 1972.

[15] N. Kikuma, Adaptive signal processing with array antenna, Science and Technology Publishing Company, 1999 (Japanese edition).

\section{Appendix}

Figures A. 1 and A. 2 show radiation patterns of the sleeve antenna and slotted cylinder antenna, respectively. These were measured in an anechoic chamber. The radiation patterns for co-polarization of both antennas are almost identical. The cross polarization discrimination (or XPD) in both $3-\mathrm{dB}$ beamwidths is better than $15 \mathrm{~dB}$ in both antennas.

Figure A. 3 shows our Rx antenna configuration. The $\mathrm{Rx}$ antenna consists of ten vertically-aligned dual-polarized sub-array antennas. Here, the top and bottom sub-array antennas are dummy antennas to make the radiation patterns of the operating sub-array antennas (\#1 - \#8) equal. The output ports of the dummy sub-array antennas are terminated, and the output ports of the operating sub-array antennas are connected to the input ports of a receiver. Each sub-array antenna consists of five printed dipole antennas, where three antennas are elements for reception of vertically polarized waves and two antennas are elements for reception of horizontally polarized waves, as shown in Fig. A. 3. Received co-polarized waves are synthesized in each sub-array antenna.

Figure A. 4 shows the radiation patterns of the 4th sub-

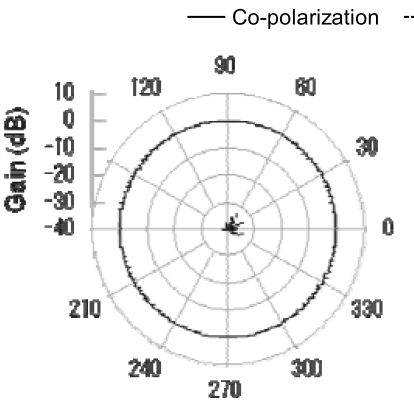

(a)

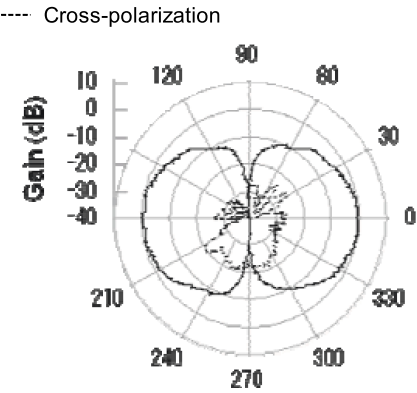

(b)
Fig. A. 1 Radiation patterns of sleeve antenna. (a) Azimuth pattern, (b) elevation pattern.

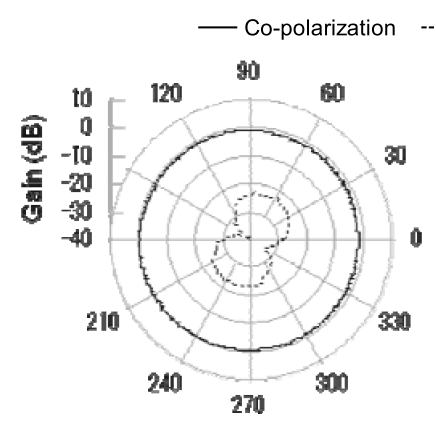

(a)

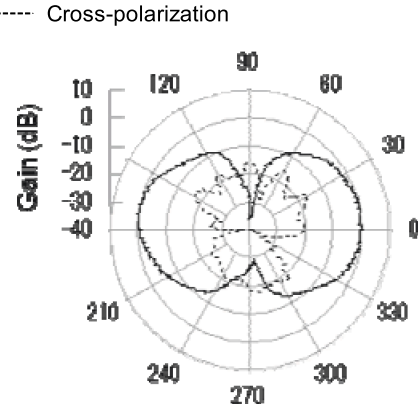

(b)
Fig. A. 2 Radiation patterns of slotted cylinder antenna. (a) Azimuth pattern, (b) elevation pattern. 


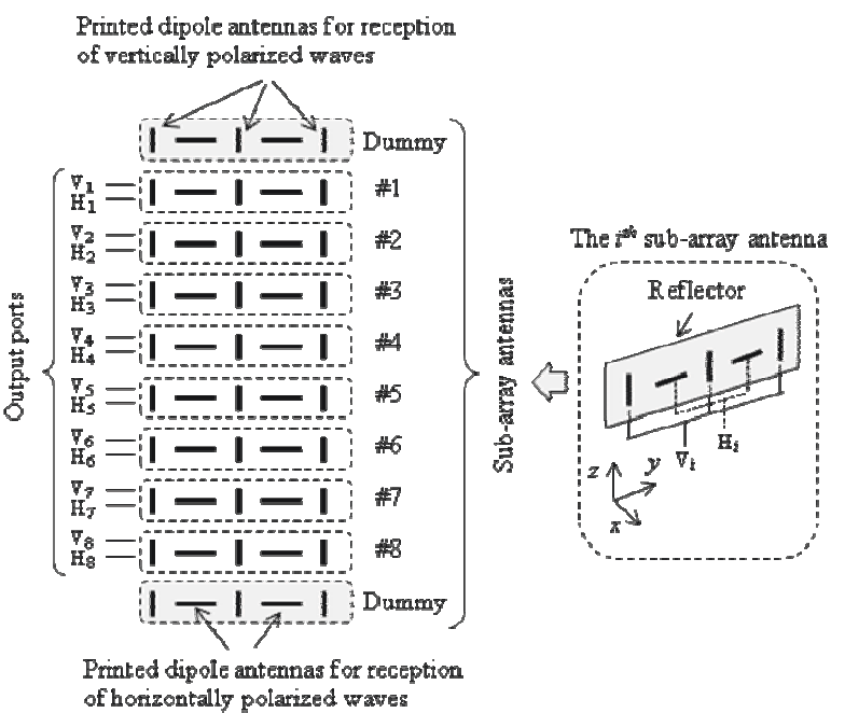

Fig. A. 3 Rx antenna configuration.

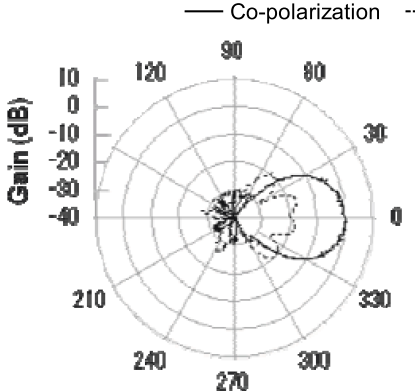

(a)

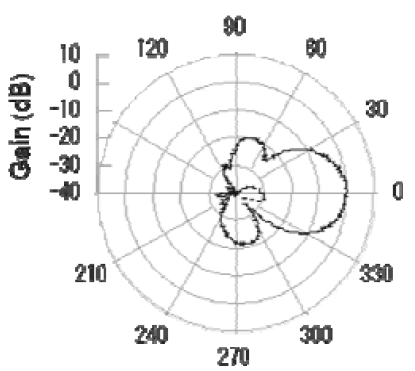

(c)

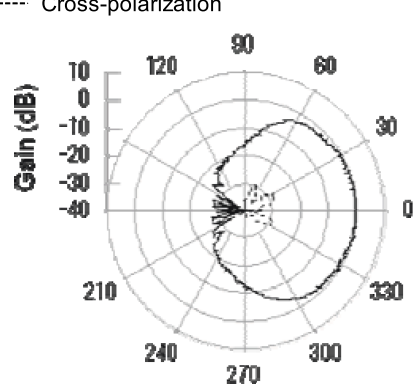

(b)

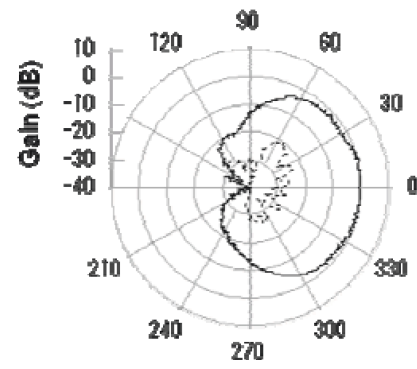

(d)

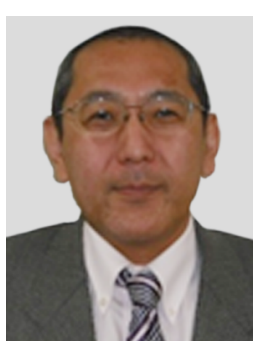

Tetsuro Imai was born in Tochigi, Japan, in 1967. He received his B.S. and Ph.D. degrees from Tohoku University, Japan, in 1991 and 2002, respectively. He joined the Wireless System Laboratories of Nippon Telegraph and Telephone Corporation (NTT), Kanagawa Japan, in 1991. Since then, he has been engaged in the research and development of radio propagation, antenna systems and system design for mobile communications. He is now Senior Research Engineer of the Research Laboratories, NTT DOCOMO, INC., Kanagawa, Japan. He received the IEICE Young Researcher's Award in 1998 and the IEICE Best Paper Award in 2006. Dr. Imai is a Member of IEEE.

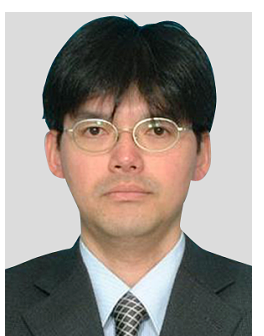

Koshiro Kitao was born in Tottori, Japan, in 1971. He received his B.S., M.S., and Ph.D. degrees from Tottori University, Tottori, Japan in 1994, 1996, and 2009, respectively. He joined the Wireless Systems Laboratories, Nippon Telegraph and Telephone Corporation (NTT), Kanagawa, Japan, in 1996. Since then, he has been engaged in the research and development of radio propagation for mobile communications. He is now Research Engineer of the Research Laboratories, NTT DOCOMO, INC.,

Fig. A. 4 Radiation patterns of the 4th sub-array antenna. (a) Azimuth pattern of $\mathrm{V}_{4}$ port, (b) elevation pattern of $\mathrm{V}_{4}$ port, (d) azimuth pattern of $\mathrm{H}_{4}$ port, (d) elevation pattern of $\mathrm{H}_{4}$ port. Here, front of the sub-array antenna is in the direction of 0 degrees.

array antenna, which were measured in an anechoic chamber. We find that the main lobe patterns for co-polarization of the $\mathrm{V}_{4}$ and $\mathrm{H}_{4}$ ports are almost identical, and the XPD in both $3-\mathrm{dB}$ beamwidths is better than $15 \mathrm{~dB}$. Note that the radiation characteristics of the other sub-array antenna are almost identical to that for the 4th sub-array antenna. 